\title{
Analysis on Purchase on Selection of Computer Hardware and System Maintenance
}

\author{
${ }^{1}$ Haiyan Dang, ${ }^{2}$ Lei Guo, ${ }^{1}$ Dianxue Shao \\ ${ }^{1}$ Mingde College, Northwestern Polytechnical University, Xi'an, 710124, China \\ ${ }^{2}$ Shaanxi Normal University, Xi'an, 710062, China
}

Keywords: computer; hardware; purchase on selection; system maintenance

\begin{abstract}
Computer system is provided with many advantages, including calculation, judgment, storage and common use. Reasonable and effective application of computer can help users to quickly finish works and live a better life. However, it is easy for computer system to have some faults in the use process. Moreover, it is easily attacked by hackers, invaded by virus, and destroyed by improper operation. Consequently, computer application effects will be reduced. To this end, the author begins with a simple summary of computer, makes an analysis on purchase on selection of computer and maintenance of computer system, and expects to be helpful for the improvement to application effect of computer system.
\end{abstract}

\section{Introduction}

With booming economy, science and technology at prevent in China, the application of computer system becomes wider and wider. It plays a great role in making it convenient for people's life and working. In spite of this, it is also easy for computer system to have some faults in the use process. Moreover, it is easily attacked by hackers and invaded by virus. Under such circumstance, vast users should emphasize reasonable selection of computer, enhance daily maintenance of computer system, and improve security and application of computer system.

\section{A Simple Summary of Computer}

Computer system is composed of two parts: hardware and software. Hardware system mainly consists of CPU, storage, input output control system and various external devices. CPU is mainly used for high-speed computation processing of information. Its processing speed can realize hundreds of millions of operations per second. Computer can be used for high-speed computation through inferior components. Storage is mainly used to store programs, data, information, files and others. With large storage space, storage can store mass data or information, making it very convenient for people to store important files or materials. Various output devices and external equipment are served as an information convertor between human and computer. Through output devices and external equipment, information can be introduced or led into computer, resulting in a remarkable improvement to applicability of computer. Computer software consists of system software, support software and application software. System software is composed of operating system, utility program, compile program and others. Operating system is used for management control over various hardware resources, so as to guarantee effective application of hardware resources. Utility program is mainly used to serve users and make it convenient for users to utilize computer to compile words. The function of compile program is to translate user's assembly language or program compiled by certain advanced language into performable machine language program. Support software consists of interface software, tool software and environmental database, which can support the environment of computer and provide software research and development tools. Application software is a specific program written by users according to specific needs. Operated by virtue of system software and support software, application software is served as the outermost layer of software system. Computer system supported by software and hardware is provided with many advantages. To be specific: 
(1) Calculation: computer can be used for all complex calculations. Moreover, it can quickly finish calculations, promoting complicated calculations to be simplified;

(2) Judgment: according to information or different circumstances, computer can make correct judgment, providing guidance to management, control, decision making and others;

(3) Storage: computer is provided with storage system with ultra-large capacity, which can be used to store mass data or files for users;

(4) Accuracy: as long as word length is enough, computer accuracy is unlimited theoretically;

(5) Speediness: the minimum unit is nanosecond for once-through operation of computer, resulting in remarkable improvement to running speed of computer;

(6) Common use: as long as practical needs are combined and computer program is reasonably operated, the application of computer can meet application needs of users. This also fully indicates that computer system is of general applicability;

(7) Ease of use: rich high-performance software and intelligent man-machine interfaces make it very convenient for use of users.

To sum up, computer system is provided with relatively strong application effect. Moreover, it is provided with many functions including calculation, judgment, programming and storage, making it very convenient for people's office works.

\section{Selection of Computer Hardware}

As an integral part of computer, hardware system is used to guarantee good quality of computer hardware, and it plays a great role in improving applicability and service life of computer. In addition, different parameters of computer hardware will result in different focuses on application of computer. Therefore, computer hardware should be selected with grate attention. For selection of computer hardware, the following items should be taken into account:

Firstly, the selection of CPU; as a core component of computer, CPU plays a decisive role in overall operation of computer. To guarantee high-speed and effective operation of computer, three indexes (number of core, buffer memory and dominant frequency) of CPU should be inspected and verified in details in the selection process, making sure that CPU is of a large number of cores, good buffer memory effect and strong dominant frequency applicability. Thus, CPU can be used for effective data calculation, for effective control over program operation and for improvement to computer performance.

Secondly, selection of memory (i.e. storage of computer); computers in the present market are of different memories. Some computers are of small memories, and some computers are of large memories. However, it is not true that greater memory is better. Excessively large memory will affect running speed of computer. Therefore, users should select the most suitable memories according to specific needs.

Thirdly, selection of hard disk; hard disk is an auxiliary storage on the premise that internal storage is used as main storage. Hard disk is applied in computer, and it is provided with many functions, including fast reading speed and large storage space. The most suitable hard disks with good application effects should be selected by combining capacity and number of revolutions of hard disks, making sure that storage performance of computer can be improved.

Fourthly, selection of display; as output device of computer, display is mainly used to serve users. Display allows users to watch materials, files and others through it. At present, there are two types of computer display in China: LCD and LED. LED display is composed of diode; while LCD display is composed of liquid crystal. The two displays differ from each other in contrast ratio, bright spot, visual angle response time and other perspectives. Therefore, contrast ratio, bright spot, visual angle response time and other perspectives should be used for reference in the selection process, so as to make the most correct decisions.

Fifthly, selection of mainboard (i.e. connecting plate); mainboard is composed of CPU slot, storage slot, various broadening slots, SATA interface, IDE interface and others. As an integral part of computer, mainboard decides applicability of computer to some extent. To guarantee effective 
application of computer for a long period, emphasis should be mainly laid to competence of support hardware and good performance of chip during selection of mainboard. Its chip is of very strong functions, and can support high-speed running of the whole computer. On this basis, mainboard should be selected with great attention.

Sixthly, selection of display card; the function of display card is connection, but it is mainly used to connect with mainboard and display. During selection of display card, size and speed of video memory should be taken as criteria. Display card should be reasonably selected, making sure that it can be effectively connected with mainboard and display, and that it can support effective application of computer.

Seventhly, selection of case; case should be compatible with total energy consumption of computer hardware and matched with the type of mainboard. Therefore, case should be selected in the last step. On the basis of the determination of mainboard and computer hardware, appropriate case should be selected. In addition, heat dissipation, appearance, safety and material of case should be also taken into account in the selection process. Cases in the present market are of different types, including business type and domestic type. Therefore, appropriate case should be selected according to specific needs. On this basis, safety, model, quality, heat dissipation, appearance and others of case should be analyzed and considered, so as to select the most desirable one.

\section{Maintenance Strategies of Computer System}

Computer system maintenance is a piece of work of great importance. It can not only guarantee effective application of computer but also improve service life of computer. On this basis, the author analyzes relevant strategies for computer system maintenance and specific measures for computer system maintenance in the next paragraphs.

(I) Strategies for computer system maintenance

Main strategies for computer system maintenance are listed as follows:

(1) Preventive maintenance: probable faults or problems of computer system should be specified by combining use information and business development trend of computer. On this basis, computer should be subject to preventive maintenance, so as to enhance safety and applicability of computer and prevent relevant problems from affecting application of computer.

(2) Corrective maintenance: it is difficult for daily simple system test to detect all problems existing in computer system. Hidden corrective errors existing in computer system should be processed by means of deep inspection, so as to find out hidden corrective errors in computer system, perform corrective maintenance for computer system, and improve application effect of computer.

(3) Adaptive maintenance: adaptive maintenance is a piece of maintenance work performed to guarantee healthy operation of computer system. In adaptive maintenance of computer system, application situation of computer system is used in combination, so as to make proper adjustment to all aspects of the whole computer, optimize all parts of computer system, and improve operation effect of computer system. Combining upgrading of current equipment and reform of network mechanism, computer system is subject to optimized maintenance, so as to make continuous improvement to deficiencies of computer system and use effect of computer. Adaptive maintenance is an effective measure to promote upgrading and development of computer system.

(II) Specific measures for computer system maintenance

Based on above strategies for computer system maintenance, specific measures are taken for computer system:

(1) Install and set antivirus software and firewall for computer

Antivirus software and firewall are effective measures for safety of computer. To prevent computer system from attacking by hackers or invaded by virus, strengthening setting of computer system firewall can effectively defense invasion of hackers or virus. Moreover, installation and setting of antivirus software can be used for all-round virus killing for computer system, preventing virus from entering computer system and thus invading the entire computer or disabling computer. Here, one point needs to be explained. That is, computer system should be subject to detailed 
understanding before installation and setting of firewall and antivirus software. On the premise of making it explicit for functions of firewall and antivirus software, firewall and antivirus software should be correctly installed so that both firewall and antivirus software can be effectively applied in computer system.

(2) Backup computer system information on a regular basis

It is difficult for any computer to avoid some unpredictable system faults in the use process. These faults will damage system files and others. Some human caused errors will also lead to loss of system data. To avoid immeasurable losses to users cased by loss of system information, it is of great significance for regular backup of computer system information. At present, Ghost software is mainly used for backup of computer system information. This software includes two backup patterns: i) Ghost data backup through light disk; and ii) backup onto computer hard disk through Ghost software. Both the two patterns can guarantee that computer system information will not result in loss of computer system information due to system fault or human caused error.

(3)Check and mend network faults on a regular basis

Checking and mending of network faults can reduce network attacks from illegal programs. At present, computer system faults are mainly checked and mended by connecting agent mode to important server host of computer. This method can be used for all-round inspection for network faults of computer system. Once any network faults are found in computer system, users will be remaindered of network maintenance and mending of network faults immediately. Thus, safe and effective application of computer is guaranteed.

\section{Conclusion}

Nowadays, computer has already become an integral part of people's life and work. It is of great importance to enhance use and maintenance of computer. Such enhancing is remarkably helpful for people's normal and effective application of computer for a long term. To realize long-term effective application of computer, it is necessary for users to refer to relevant parameters for reasonable selection of computer mainboard, case, display, hard disk, storage and others, so as to guarantee that selected computer hard disk can support high-speed operation of computer. Besides, computer system maintenance is also very important. Computer firewall and antivirus software should be installed and set as well. Moreover, users should also regularly backup computer system information, check and mend network faults, or take other measures to improve security and applicability of computer system. With continuous development of science and technology in China, I believe that computer system will be continuously optimized and innovated, and used to serve users in a better way.

\section{References}

[1] Jiang Wei, Selection of Computer Hardware and System Maintenance [J], Practical Electronics, 2013 (24);

[2] Zhou Rui, Analysis on Selection of Computer Hardware and System Maintenance Issues [J], Computer CD Software and Application, 2014 (03);

[3] Jia Jinlou and Huang Guoming, Lighting Prevention Detection Elements of Computer Information System [A], Weather, Climate and Sustainable Development - collected papers of Henan Meteorological Society on annual meeting in 2010, [C], 2010;

[4] Huang Leming, Chen Gang and Li Ming, Surge Protection in Computer System Lighting Prevention Project Design [A], collected academic papers on the $3{ }^{\text {rd }}$ Hubei Science, Technology and Weather Branch Forum and 2005 Academic Annual Conference of Hubei Meteorological Society [C], 2005;

[5] Yu Jie and Yang Honglei, Computer Packaging \& Commissioning and Troubleshooting [A], collected papers in 2009 annual meeting of Tianjin Television Technology Research Institute (1) [C], 
2009;

[6] Gao Yin and Liu Shengjie, Analysis on Common External Faults and Maintenance of Computer - Discussion from Hardware [J], Agriculture Network Information, 2006 (06);

[7] Zhang Yongbin, Li Linhong and Shi Ziyuan, Discussion on Management and Maintenance of Computer Lab in Colleges and Universities [A], collected papers in 2007 laboratory work seminar of Beijing Higher Education Association [C], 2007;

[8] Qiao Renlu, Discussion on Management and Maintenance of Computer Lab [J], Technology and Market, 2009 (08);

[9] Wang Xuehai and Su Baozhi, Computer System Security Theory and Its Realization [A], collected papers of the $3^{\text {rd }}$ Computer Security Technical Seminar [C], 2002. 